\title{
Motion Planning For Steerable Needles in 3D Environments with Obstacles Using Rapidly-Exploring Random Trees and Backchaining
}

\author{
Jijie $\mathrm{Xu}^{1}$, Vincent Duindam ${ }^{2}$, Ron Alterovitz ${ }^{1,3}$, and Ken Goldberg ${ }^{1,2}$ \\ 1. Department of IEOR, University of California, Berkeley, USA \\ 2. Department of EECS, University of California, Berkeley, USA \\ 3. Comprehensive Cancer Center, University of California, San Francisco, USA
}

\begin{abstract}
Steerable needles composed of a highly flexible material and with a bevel tip offer greater mobility compared to rigid needles for minimally invasive medical procedures. In this paper, we apply sampling-based motion planning technique to explore motion planning for the steerable bevel-tip needle in 3D environments with obstacles. Based on the Rapidly-exploring Random Trees (RRTs) method, we develop a motion planner to quickly build a tree to search the configuration space using a new exploring strategy, which generates new states using randomly sampled control space instead of the deterministically sampled one used in classic RRTs. Notice the fact that feasible paths might not be found for any given entry point and target configuration, we also address the feasible entry point planning problem to find feasible entry points in a specified entry zone for any given target configuration. To solve this problem, we developed a motion planning algorithm based on RRTs with backchaining, which grow backward from the target to explore the configuration space. Finally, simulation results with a approximated realistic prostate needle insertion environment demonstrate the performance of the proposed motion planner.
\end{abstract}

\section{INTRODUCTION}

Inserting a needle to deliver treatment or to biopsy tissue is a minimally invasive and inexpensive percutaneous procedure that can often be performed on an outpatient basis. Achieving accuracy in the needle tip position is challenging due to lack of maneuverability, limited visibility, and possible obstructions between the needle entry point and the target zone. As an alternative to the traditional rigid symmetric-tip needle, collaborators at Johns Hopkins University and the University of California, Berkeley are developing a new class of highly flexible, bevel-tip needles that offer improved mobility, enabling them to reach previously inaccessible targets while avoiding sensitive or impenetrable areas, such as the urethra and the penile bulb around the prostate as illustrated in Fig. 1 [1], [2].

Motion planning for bevel-tip steerable needle has been studied in the two-dimensional image plane [3], [4]. Planning motions for steerable needle in 3-D environment is more difficult due to the nonholonomic constraint and the underactuation inherent in the bevel-tip design. Motion of the bevel-tip needle in a 3D workspace is controlled by only two degrees of freedoms at the needle base: insertion along the needle axis and rotation about the needle axis. Asymmetric forces on the needle's beveled tip cause the needle to bend and follow a curved path through the tissue, and the needle tip's

This work was supported by NIH grant R01 EB006435 and the Netherlands Organization for Scientific Research.

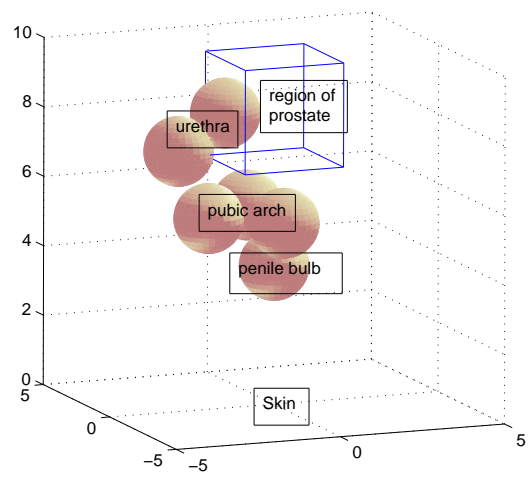

Fig. 1. An approximation of 3D environment of needle insertion for prostate using spherical obstacles.

orientation changes during insertion. The rotation not only changes the needle tip's orientation about its axis, but also navigates the direction of the insertion. More flexible rotations have to be made by the needle in order to generate its path in the $3 \mathrm{D}$ workspace. This makes motion planning for the beveltip steerable needle in 3D environments more complicated.

In this paper, we apply sampling-based motion planning technique to explore motion planning of the bevel-tip steerable needle in 3D environments with obstacles. We develop a new motion planner by inspired by the well-known rapidlyexploring random trees method (RRTs). In order to make a trade-off between the complexity and completeness of the RRT exploration, we propose a new vertex generation strategy by using a randomly sampled control space instead of the deterministically sampled one. Considering the requirements of real clinical tasks, we further address the feasible entry point planning problem, and solve it by developing a planner based on RRTs with backchaining. To the best of our knowledge, this work is the first to apply RRT-based motion planning techniques in steerable needle motion planning in $3 \mathrm{D}$ environments.

\section{RELATED WORK}

The bevel-tip needle design has been shown to significantly affect the needle bending forces during insertion [5]. Based on this observation, Webster et al. [6], [7], [8] experimented further and showed that steerable bevel-tip needles follow 
paths of constant curvature in the direction of the bevel tip. They also developed a nonholonomic model of steerable beveltip needle motion in stiff tissues based on a generalization of the bicycle model and fit model parameters using experiments with tissue phantoms [7].

Motion planning for steerable needles in a 2D workspace has been studied, incorporating the effects of tissue deformations and motion uncertainty into planning. Modeling the bevel-tip needle's motion in a 2D workspace as a nonreversible Dubins car, Alterovitz et al. formulated the 2D steerable needle motion planning problem as a nonlinear optimization problem that uses a simulation of tissue deformation during needle insertion as a function in the optimization [2]. To consider motion uncertainty due to needle/tissue interaction, Alterovitz et al. formulated the motion planning problem as a Markov Decision Process (MDP) using a discretization of the space and orientations [3], [4] and using the Stochastic Motion Roadmap (SMR), a sampling-based approach [9]. Alterovitz et al. also introduced a motion planner to solve for the optimal insertion location in 2-D [4], a problem we consider in this paper for 3D environments.

With the development of volumetric medical imaging techniques, research on steerable needle insertion has been extended to more complex 3D environments. Kallem et al. [10] developed a nonlinear controller to stabilize the needle's 3D motion on a desired 2D plane for use with 2D imaging modalities and motion planning algorithms. Park et al. [11] treated the kinematics of the bevel-tip needle as the 3D extension of the standard unicycle model, and proposed a diffusion-based motion planning method to numerically compute a path in the obstacle-free stiff tissue. Abolhassani et al. [12] proposed a method to minimize the needle's deflection by controlling the needle's rotation during the insertion, using online measurements through force/moment sensing. By representing the motion of the bevel-tip needle as a screw motion in a 3D environment, Duindam et al. [13] formulated 3D motion planning of the steerable needle as a dynamical optimization problem with a discretization of the control space. We study a similar problem of finding valid needle paths in 3D environments with obstacles, yet our approach builds a global roadmap that (probabilistically) explores the entire workspace, whereas the previous algorithm [13] only considered locally optimal paths and may fail in more complex environments.

The Rapidly-exploring Random Tree (RRT) has shown its potential in dealing with motion planning problems for nonholonomc systems [14][15]. It incrementally grows a tree toward the target configuration by searching feasible paths in the configuration space, and provides an efficient and quick search in complex environments of high dimensions with different constraints [14], [16], [17], [18]. By alternating between growing two trees (rooted at the start and goal configuration respectively) towards random samples and towards each other, Kuffner et al. developed the bidirectional RRT Connect algorithm to increase the efficiency [19]. Branicky et al. extended the RRT-based method to solve motion planning problems in systems with a hybrid configuration space and constraints
[20]. By using hints obtained from obstacles to navigate the randomly sampled nodes away from obstacles, Rodriguez et al. developed an obstacle-based RRT method to efficiently explore the tree in difficult regions in the $\mathcal{C}$-space [21]. These works developed different exploration strategies for RRTs with randomly sampled $\mathcal{C}$ space and deterministic control space. Knepper et al. experimentally studied the relationship between path sampling strategy and mobile robot performance, and showed that different deterministic samplings of path sets led to different performances of motion planners for mobile robots [22]. In this paper, we propose an exploration strategy for the RRT with both randomly sampled $\mathcal{C}$-space and control space. To the best of our knowledge, this is the first paper that applies RRT-based method to the steerable needle motion planning and develops a unidirectional exploration strategy using a randomly sampled control space. Bidirectional exploration with sampled control space will be explored in future work.

\section{Problem Statement}

To make the problem well defined, we make the following assumptions:

1). The bevel-tip needle is rigid, and rotating the needle at the base will not change its position in the workspace.

2).The needle body follows motion of the needle tip, and the tip's orientation exactly follows the base's orientation.

3). The feasible workspace is stiff and defined as a 3D cuboid. No deformation of the workspace and obstacles is considered in this paper.

4). Obstacles are 3D balls with constant radius. Obstacles with more complicated shapes will be considered in future work. With the above assumptions, the steerable needle motion planning problem can be stated as follows.

Problem 1 (Steerable needle motion planning): Given an initial configuration and a target zone, determine a feasible path and the corresponding sequence of controls (insertion depths and rotations at the needle base) so that the needle tip reaches the target zone from the initial configuration while avoiding obstacles and staying inside the workspace.

Input: Boundaries of the workspace, parameters of the beveltip needle, locations and radius of the spherical obstacles, an entry configuration of the needle, a target zone that the needle is required to reach.

Output: A sequence of discrete controls, with which the needle $\overline{\text { steered }}$ from the given entry point to reach the target zone, or a report that no path is found.

Because of the needle's nonholonomic constraints and the structure of the environment, there may not exist feasible paths reaching the target for all given initial configurations. Moreover, feasible paths for any given initial configuration may not be found by motion planners developed for Problem 1 . For this reason, we address the feasible entry point planning problem as follows.

Problem 2 (Feasible entry point planning): Given a specified target configuration and an initial zone, determine a feasible entry point in the zone and the corresponding sequence of controls (insertion depths and rotations at the needle's base) 


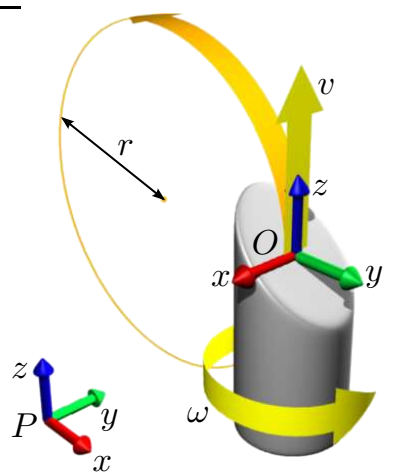

Fig. 2. Model of the bevel-tip needle.

so that the needle tip reaches the specified target from this entry point while avoiding obstacles and staying inside the workspace.

Input: Boundaries of the workspace, parameters of the beveltip needle, locations and radius of the spherical obstacles, the target configuration, the entry zone.

Output: A feasible entry point and the corresponding sequence of discrete controls, with which the needle reach the target region, or a report that no path is found.

\section{Kinematics of the BeVel-Tip Flexible NeEdle}

Consider the bevel-tip needle shown in Fig. 2. Referring to the notations in [23], attach a spatial frame $P$ to the base of the needle and a body frame $O$ to the geometric center of the needle's bevel-tip, respectively. The configuration of the needle tip can be represented homogeneously by the 4 by 4 transformation matrix of the object frame relative to the spatial frame as

$$
g_{P O}=\left[\begin{array}{cc}
R_{P O} & p_{P O} \\
0 & 1
\end{array}\right] \in S E(3),
$$

where $R_{P O} \in S O(3)$ is the rotation matrix and $p_{P O} \in T(3)$ is the position of frame $O$ relative to frame $S$.

The motion of the needle is fully determined by two motions performed at the bevel-tip: insertion with velocity $v(t)$ in the $z$ direction and rotation with velocity $\omega(t)$ along the $z$ axis of the body frame $O$ [7], [13]. It has been experimentally shown by Webster et al. [8] that the bevel-tip needles will follow a constantly curved path with curvature $\kappa=\frac{1}{r}$ when pushed with zero bevel rotation velocity, i.e. $\omega=0$. The instantaneous velocity of the needle tip can be represented in the body frame $O$ as

$$
V_{P O}^{b}=\left[\begin{array}{ll}
\mathbf{v}^{T} & \mathbf{w}^{T}
\end{array}\right]^{T}=\left[\begin{array}{llllll}
0 & 0 & v(t) & v(t) / r & 0 & \omega(t)
\end{array}\right]^{T} .
$$

When $V_{P O}^{b}$ is constant, i.e., $v(t)$ and $\omega(t)$ are constant, the configuration of the needle tip relative to the spatial frame after being pushed for a time interval $t$ is

$$
g_{P O}(t)=g_{P O}(0) e^{\hat{V}_{P O}^{b} t},
$$

where $g_{P O}(0)$ is the initial configuration of the needle frame relative to the spatial frame, and

$$
\hat{V}_{P O}^{b}=\left[\begin{array}{cccc}
0 & -\omega(t) & 0 & 0 \\
\omega(t) & 0 & -v(t) / r & 0 \\
0 & v(t) / r & 0 & v(t) \\
0 & 0 & 0 & 0
\end{array}\right] .
$$

For constant $V_{P O}^{b}$, the needle motion can also be interpreted as a screw motion with constant axis and pitch [23], [13]. When the entire insertion of the needle is discretized into $N$ steps with corresponding $N$ time segments $\left\{I_{1}, \cdots, I_{N}\right\}$, and the velocity $V_{P O}^{b}\left(I_{n}\right)$ is fixed in each step, the final configuration of the needle tip can be computed as a product of exponentials

$$
g_{P O}(T)=g_{P O}(0) e^{\hat{V}_{P O}^{b}\left(I_{1}\right) I_{1}} \cdots e^{\hat{V}_{P O}^{b}\left(I_{N}\right) I_{N}} .
$$

\section{Motion Planning for Steerable Needle using FORWARD RRTS}

The configuration of the needle tip can be represented by its position $(x, y, z)$ and Euler angles $(\phi, \theta, \psi)$. Since the insertion task only requires the needle to reach a target position inside the 3D workspace, the configuration space of the motion planning is equivalent to $\mathbb{R}^{3}$. Given boundaries of the workspace $\left(\left[x_{\min }, x_{\max }\right],\left[y_{\min }, y_{\max }\right],\left[z_{\min }, z_{\max }\right]\right)$, locations of the obstacles, the needle's initial configuration $s_{\text {init }}$ and target zone $S_{\text {goal }}$, a tree can be constructed with the classic RRT [17].

Algorithm 1: (Forward RRT with deterministic control space sampling) Initialize a tree $\mathcal{T}$ rooted at $s_{\text {init }}$. For a randomly sampled collision free state $s_{\text {rand }}$ in $\mathcal{C} \mathcal{S}_{\text {free }}$, we search $\mathcal{T}$ for the nearest neighbor of $s_{\text {rand }}$, denoted by $s_{\text {near. }}$. By applying deterministically sampled control inputs to $s_{\text {near }}$ for a short time increment $\delta t$, we generate a set of all possible new states $S_{\text {new }}$. In $S_{\text {new }}$, the nearest neighbor of $s_{\text {rand }}$, denoted by $s_{\text {new }}$, is found and added to $\mathcal{T}$. Such an exploration is repeated until $\mathcal{T} \cap S_{\text {goal }} \neq \emptyset$ or the number of iteration reaches its limit.

The distance used in the nearest neighbor search can be defined in different ways by defining different metrics on the configuration space. To let the RRT grow toward the target zone fast, we apply a biased distribution of the sampling states in $\mathcal{C} \mathcal{S}_{\text {free }}$. The state $s_{\text {rand }}$ is sampled mostly uniformly inside the boundaries of the configuration space, except for a higher density in $S_{\text {goal }}$. If $s_{\text {rand }}$ collides with any obstacle, it is discarded and new states are sampled until one in $\mathcal{C} \mathcal{S}_{\text {free }}$ is found.

The path of the bevel-tip needle can only follow curved paths with a minimum curvature $\kappa=1 / r$, as shown in Fig. 2 . For this reason, configurations that can be reached by the needle are locally constrained to be inside the volume of a crateriform region (see Fig. 3) defined locally by

$$
p_{z} \geq \sqrt{2 r \sqrt{p_{x}^{2}+p_{y}^{2}}-p_{x}^{2}-p_{y}^{2}}
$$

with $\left(p_{x}, p_{y}, p_{z}\right)$ the coordinates of a point in body frame $O$.

Algorithm 1 requires a deterministic sampling of the control space, whose resolution greatly affects the performance of the planning algorithm.[16]. A higher resolution leads to a 


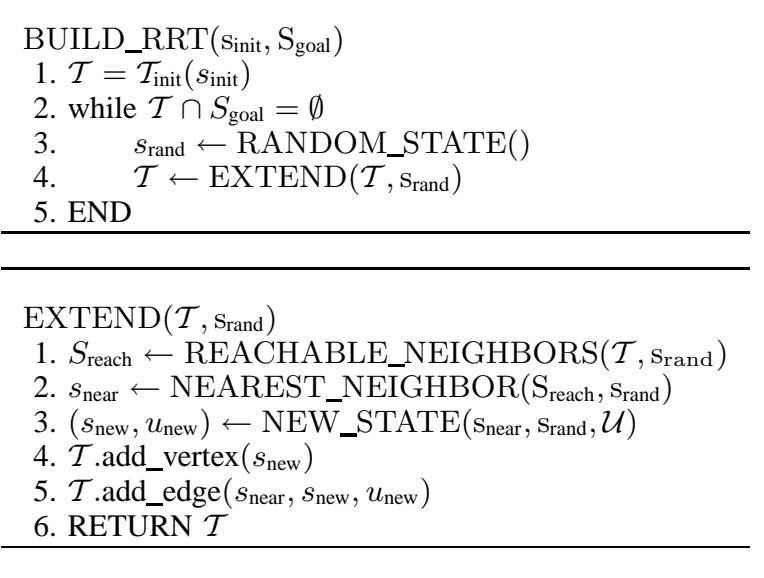

REACHABLE_NEIGHBORS $(\mathcal{T}$, Srand $)$

1. For all $s_{i} \in \overline{\mathcal{T}}$

2. if $s_{\text {rand }}$ is reachable from $s_{i}$

3. add $s_{i}$ to $S_{\text {reach }}$

4. RETURN $S_{\text {reach. }}$.

NEW_STATE $\left(\right.$ snear $_{\text {n }}$ sand, $\left.\mathcal{U}\right)$

1. $\mathcal{U}_{\text {rand }} \leftarrow$ CONTROL_SAMPLING $(\mathcal{U})$

2. FOR all $u_{i} \in \mathcal{U}_{\text {rand }}$

3. $s_{\text {new }}(i)=s_{\text {near }}+F_{q_{\text {near }}}\left(s, u_{i}\right) \delta t$

4. $S_{\text {new }}=\cup_{i} s_{\text {new }}(i)$

5. $s_{\text {new }} \leftarrow$ NEAREST_NEIGHBOR $\left(\mathrm{S}_{\text {new }}, \mathrm{S}_{\text {rand }}\right)$

6. $u_{\text {new }}=u_{i}$ such that $s_{i}=s_{\text {new }}$

7. RETURN $s_{\text {new }}, u_{\text {new }}$

TABLE I

RAPIDLY-EXPLORING RANDOM TREES BASED PLANNER WITH CONTROL SPACE SAMPLING.

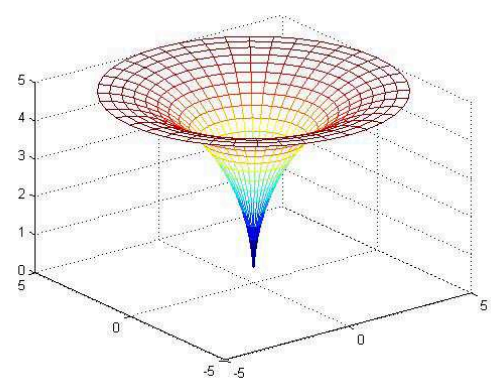

Fig. 3. The crateriform reachable region of local needle motion.

more detailed exploration with more complexity, but a lower resolution leads to a fast exploration with less information on the connectivity and structure of the free space. Instead of using the deterministic discretization of the control space, we sample a set of control inputs uniformly in the control space, using CONTROL_SAMPLING(), within a predefined range $\left[v_{\min }, v_{\max }\right] \times\left[\omega_{\min }, \omega_{\max }\right]$, and apply all sampled control inputs to $s_{\text {near }}$ for $\delta t$ to generate the set of possible new states $S_{\text {new }}$. By doing so, we not only explore the RRT toward all possible directions with same probability, but also extend the RRT toward the sampled states by various depth with same probability. With such an exploration strategy, an RRT can be constructed using the following algorithm.

Algorithm 2: (Forward RRT with random control space sampling) Initialize the tree $\mathcal{T}$ rooted at $s_{\text {init }}$ and randomly sample a collision free state $s_{\text {rand }}$ in $\mathcal{C} \mathcal{S}_{\text {free }}$. A reachable neighbor search is applied to find a set of states $S_{\text {reach }}$, from which $s_{\text {rand }}$ can be reached. After finding $s_{\text {near }} \in \mathcal{T}$, which is the nearest neighbor of $s_{\text {rand }}$, we uniformly sample the control space and apply all sampled control inputs to $s_{\text {near }}$ for $\delta t$ to generate a set of possible new states $S_{\text {new }}$. The nearest neighbor of $s_{\text {rand }}$ in $S_{\text {new }}$ is found as $s_{\text {new }}$ and added to the tree. Such exploration is repeated until $\mathcal{T} \cap S_{\text {goal }} \neq \emptyset$ or the number of iteration reaches its limit.

The scenario of Algorithm 2 is shown in Table. I. By growing the RRT with randomly sampled control inputs, Algorithm 2 probabilistically makes a trade-off between the complexity and the completeness of the exploration.

\section{Entry Point Planning for Steerable Needle USING RRTS WITH BACKCHAINING}

Configuration of the needle tip following the reversed trajectory for constant $V_{P O}^{b}$ can be represented as

$$
g_{P O}(t-\delta t)=g_{P O}(t) e^{-\hat{V}_{P O}^{b} \delta t} .
$$

A path starting from the goal configuration can be described as a reverse path starting from the entry point with the negative control space. Given the target configuration $s_{\text {goal }}$ and the specified entry zone $S_{\text {entry }}$, Problem 2 can be solved using the following algorithm.

Algorithm 3 (RRT with backchaining): Initialize the tree $\mathcal{T}$ rooted at $s_{\text {goal }}$ and randomly sample a collision free state $s_{\text {rand }}$ in $\mathcal{C} \mathcal{S}_{\text {free }}$. For any $s_{\text {rand }}$, a reachable neighborhood $S_{\text {reach }}$ is computed. After finding $s_{\text {near }} \in \mathcal{T}$, which is the nearest neighbor of $s_{\text {rand }}$, we uniformly sample the negative control space $-\mathcal{U}$ and apply all sampled control inputs to $s_{\text {near }}$ for $\delta t$ to generate a set of possible new states $S_{\text {new }}$. The nearest neighbor of $s_{\text {rand }}$ in $S_{\text {new }}$ is found as $s_{\text {new }}$ and added to the tree. Such exploration is repeated until $\mathcal{T} \cap S_{\text {entry }} \neq \emptyset$ or the number of iterations reaches its limit.

\section{Simulation Results}

We implement the proposed RRT based motion planning method for the steerable needle insertion in a 3D environments with obstacles. Since we assume that the needle is to be inserted from outside of the tissue, we only consider workspace with positive $z$-axis. The workspace is defined to be a cubical region with coordinates $(-5,5) \times$ $(-5,5) \times(0,10)$, and we use six unit-radius spherical obstacles as shown in Fig. 1, which are centered at the positions $(0,0,4),(-1.5,0,8.5),(-2.9,0,7.5),(-2,0,5.5)$, $(-0.3,1.4,5.5)$ and $(-0.3,-1.4,5.5)$, to approximate obstacles around real prostate, such as the urethra, the penile bulb and the pubic arch. The maximal number of iterations is 10000. Simulations are run on a laptop with Intel Centrino ${ }^{\circledR}$ $1.66 \mathrm{MHz}, 1 \mathrm{~GB}$ memory, and Microsoft ${ }^{\circledR}$ Windows $\mathrm{XP}^{\circledR}$ operation system. 

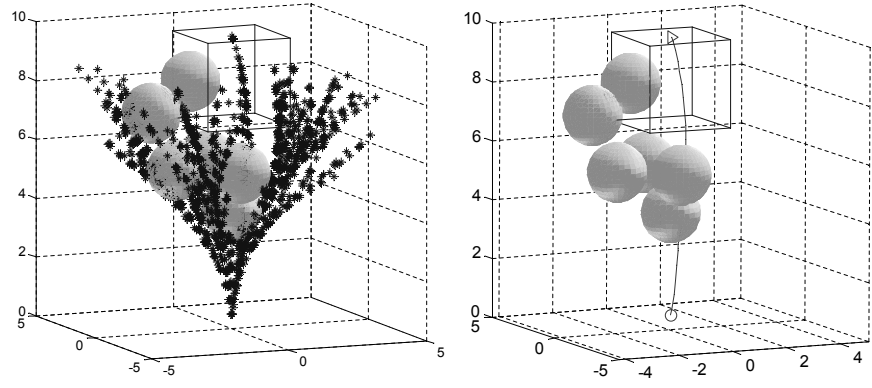

Fig. 4. Algorithm 1: (a) The exploration of the basic RRT; (b) The feasible path found by the basic RRTs method.
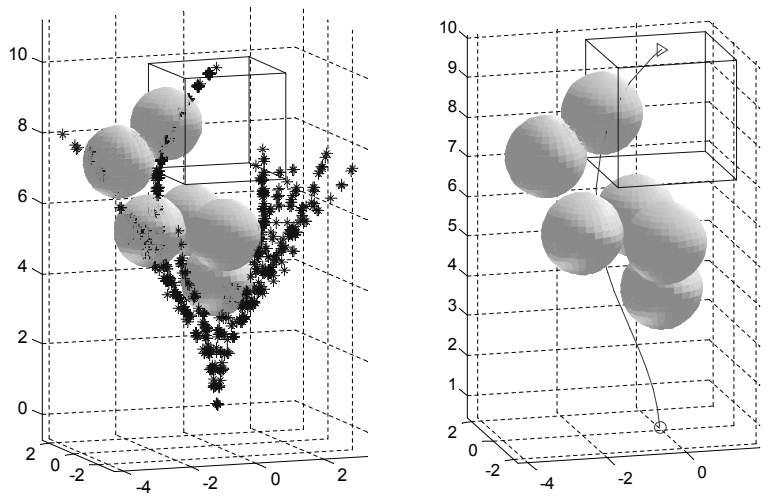

Fig. 5. Algorithm 2: (a) Exploration of the forward RRTs with control space sampling; (b) Feasible path found by forward RRTs with control space sampling.

First, we implement Algorithms 1 and 2 to solve Problem 1 for an insertion task from entry point $(0,0,0)$ with orientation $(0,0,0)$ to reach the target zone which is a ball located at $(0,0,10)$ with radius 0.01 . The range of the control inputs are defined by insertion depth in $[0.1,0.5]$ and rotation angle in $[0,2 \pi]$, and the grid size of control space deterministic sampling is 0.1 . Totally 10 trials have been done with both Algorithms 1 and 2. With Algorithm 1, 5 trials successfully found feasible paths within 10000 iterations. The average number of iterations for the RRT to reach the target region with deterministic control space sampling is 1798.5 , with the minimum at 148 and the maximum at 3500 . The average CPU time used is 1851.8 second. Fig. 4(a) shows the exploration of one of the basic RRTs with 2362 iterations, and Fig. 4(b) shows the feasible path found with this RRT, which finally reached the position at $(0.021,0.023,9.95)$.

With Algorithm 2, control inputs are uniformly sampled with insertion depth in $[0.1,0.5]$ and rotation angle in $[0,2 \pi]$. All trials successfully found feasible paths. The average number of iterations that the RRT with random control space samplings take to reach the target region is 1339.3 , with the minimum at 142 and the maximum at 3748 . The average CPU time used is 621.4 second. Fig. 5(a) shows the feasible path found by this motion planner, which finally reached the

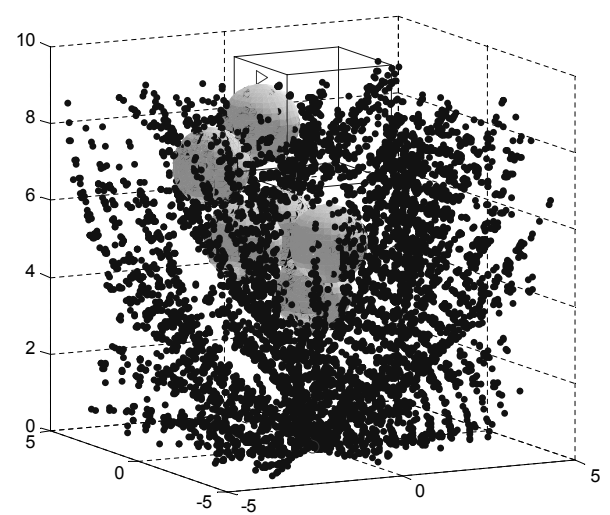

Fig. 6. Algorithm 2: Exploration of the forward RRT for a difficult target.

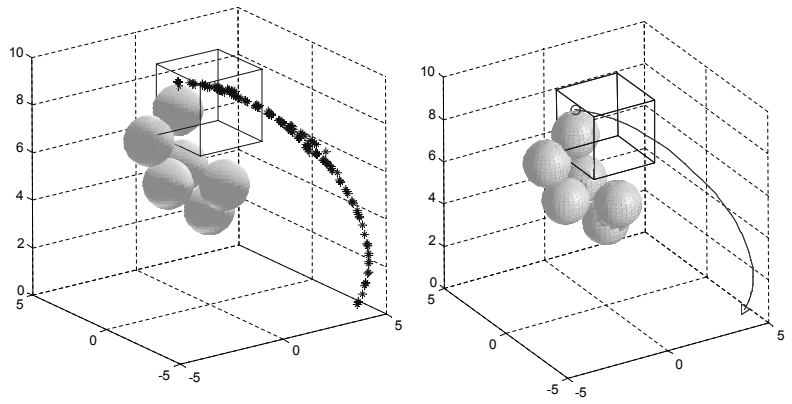

Fig. 7. Algorithm 3: (a) Exploration of one RRT with backchaining; (b) Feasible path found by the RRT with backchaining.

position at $(-0.006,0.011,9.991)$, and Fig. 5(b) shows the RRT exploration with Algorithm 2 in the free space.

Second, we consider insertion task in the same environment, and the target configuration is set to be $(-1.5,0,9.7)$. Since the target is very close to one of the obstacles and the obstacle is in the middle of the way between the entry point and the target zone, it is difficult to find a feasible path for this task. We first formulate it as Problem 1 and implement Algorithm 2 to solve it. The entry configuration is with position $(0,0,0)$ and orientation $\left(\left[-\frac{\pi}{2}, \frac{\pi}{2}\right],\left[-\frac{\pi}{2}, \frac{\pi}{2}\right], 0\right)$, and the target zone is a ball located at $(-1.5,0,9.7)$ with radius 0.001 . Totally, 5 trials have been done, but none of them can find a feasible path in 10000 iterations. Fig. 6 shows one of the explorations of such RRTs. Then we formulate it as Problem 2 and implement Algorithm 3 to solve it. The same target configuration is used and the entry region is the $x-y$ plane with $z=0$. Totally 5 trials have been done, and all

\begin{tabular}{|l|c|c|}
\hline Algorithm & 1 & 2 \\
\hline Number of trials & 10 & 10 \\
\hline Number of successes & 5 & 10 \\
\hline Average Number of iterations & 1798.5 & 1339.3 \\
\hline Average CPU time (s) & 1851.8 & 621.4 \\
\hline
\end{tabular}

TABLE II

Performance of Algorithms 1 AND 2 FOR SOlving Problem 1. 
successfully find feasible entries points and paths. The average number of iterations that the RRT with backchaining uses to find a feasible entry point is 279.6 with a minimum at 112 and a maximum at 638 , and the average CPU time used is 195.2 second. Fig. 7(a) shows exploration of one of the RRTs in the free space, and Fig. 7(b) shows the feasible path found by this with backchaining.

\begin{tabular}{|l|c|c|}
\hline Algorithm & 2 & 3 \\
\hline Number of trials & 5 & 5 \\
\hline Number of successes & 0 & 5 \\
\hline Average Number of iterations & 10000 & 279.2 \\
\hline Average CPU time (s) & N/A & 195.2 \\
\hline
\end{tabular}

TABLE III

Performance of Algorithm 2 AND 3 FOR A DifFicult TARget CONFIGURATION.

\section{CONCLUSIONS AND FUTURE WORK}

In this paper, we proposed a novel RRT-based motion planning method for bevel-tip steerable needle in 3D environment. This method used randomly sampled control space instead of the classic deterministic one to explore a tree in the workspace, which probabilistically makes a trade-off between exploration complexity and exploration completeness. We also addressed feasible entry point planning problem and proposed a method using RRT with backchaining to solve this problem. This algorithm provides a quick search toward the entry zone in $\mathcal{C}$-space with its RRT structure. Although no entry point could be found within limited iterations for some very difficult goal configuration, it can easily find feasible entry points and corresponding paths for most goal configurations because the entry zone is much less constrained. Finally, we provided simulations to explore performance of the proposed motion planners.

In this paper, we only consider motion planning for steerable needle in stiff 3D environment with spherical obstacles. In future work, we will explore this problem in deformable environments with obstacles of more complex shapes. Also, we will consider insertion tasks whose initial and goal conditions are both specified by a zone in the configuration space. Moreover, another important factor of the 3D steerable needle motion planning, uncertainties in sensing and motion, will be taken into account in future work too.

\section{ACKNOWLEDGEMENT}

We thank Allison Okamura, Greg Chirikjian, Noah Cowan, Kyle Reed, and Vinutha Kallem for their valuable assistance to this work.

\section{REFERENCES}

[1] Robert J. Webster III, Jin Seob Kim, Noah J. Cowan, Gregory S. Chirikjian, and Allison M. Okamura. Nonholonomic modeling of needle steering. International Journal of Robotics Research, 25:509-525, May 2006.

[2] R. Alterovitz, A. Lim, K. Goldberg, G. S. Chirikjian, and A. M. Okamura. Steering flexible needles under markov motion uncertainty. In Proceedings of IEEE/RSJ International Conference on Intelligent Robots and Systems, pages 120-125, 2005.
[3] R. Alterovitz, K. Goldberg, and A. M. Okamura. Planning for steerable bevel-tip needle insertion through $2 \mathrm{~d}$ soft tissue with obstacles. In Proceedings of IEEE International Conference on Robotics and Automation, pages 1640-1645, 2005.

[4] R. Alterovitz, M. Branicky, and K. Goldberg. Constant-curvature motion planning under uncertainty with applications in image-guided medical needle steering. In Proceedings of workshop on the Algorithmic Foundations of Robotics, 2006.

[5] M. D. O'leary, C. Simone, T. Washio, K. Yoshinaka, and A. M. Okamura. Robotic needle insertion: Effects of friction and needle geometry. In Proceedings of IEEE International Conference on Robotics and Automation, 2003.

[6] R. J. Webster III, N. J. Cowan, G. Chirikjian, and A. M. Okamura. Nonholonomic modeling of needle steering. In Proceedings of 9th International Symposium on Experimental Robotics, 2004.

[7] R. J. Webster III, J. Memisevic, and A. M. Okamura. Design consideration for robotic needle steering. In Proceedings of IEEE International Conference on Robotics and Automation, 2005.

[8] R. J. Webster III, J. S. Kim, N. J. Cowan, G. S. Chirikjian, and A. M. Okamura. Nonholonomic modeling of needle steering. International Journal of Robotics Research, 5/6:509-525, May, 1996.

[9] Ron Alterovitz, Thierry Siméon, and Ken Goldberg. The Stochastic Motion Roadmap: A sampling framework for planning with Markoy motion uncertainty. In Robotics: Science and Systems, 2007.

[10] V. Kallen and N. J. Cowan. Image-guided control of flexible beveltip needles. In Proceedings of IEEE International Conference on Robotics and Automation, pages 3015-2020, 2007.

[11] W. Park, J. S. Kim, Y. Zhou, N. J. Cowan, A. M. Okamura, and G. S. Chirikjian. Diffusion-based motion planning for a nonholonomic flexible needle model. In Proceedings of IEEE International Conference on Robotics and Automation, pages 4611-4616, 2005.

[12] N. Abolhassani, R. V. Patel, and F. Ayazi. Minimization of needle deflection in robot-assisted percutaneous therapy. The Internal Journal of Medical Robotics and Computer Assisted Surgery, 3:140-148, 2007.

[13] V. Duindam, R. Alterovitz, S. Sastry, and K. Goldberg. Screw-based motion planning for bevel-tip flexible needles in $3 \mathrm{~d}$ environments with obstacles. In Proceedings of IEEE International Conference on Robotics and Automation, 2008.

[14] S. M. LaValle. Rapidly-exploring random trees: a new tool for path planning. TR 98-11, Department of Computer Science, Iowa State University, 1998.

[15] S. M. LaValle. Planning Algorithms. Cambridge University Press, Cambridge, U.K., 2006. Available at http://planning.cs.uiuc.edu/.

[16] S. M. LaValle and J. J. Kuffner. Randomized kinodynamic planning. In Proceedings IEEE International Conference on Robotics and Automation, pages 473-479, 1999

[17] S. M. LaValle. Robot motion planning: A game-theoretic foundation. Algorithmica, 26(3):430-465, 2000.

[18] J. J. Kuffner. Effective sampling and distance metrics for 3D rigid body path planning. In Proceedings IEEE International Conference on Robotics \& Automation, 2004.

[19] J. Kuffner and S. LaValle. Rrt-connect: An efficient approach to singlequery path planning. In Proceedings of IEEE International Conference on Robotics and Automation, 2000.

[20] M. S. Branicky, M. M. Curtiss, J. Levine, and S. Morgan. RRTs for nonlinear, discrete, and hybrid planning and control. In Proceedings IEEE Conference Decision \& Control, 2003.

[21] S. Rodriguez, X. Tang, J. Lien, and N. Amato. An obstacle-based rapidly-exploring random tree. In Proceedings of IEEE International Conference on Robotics and Automation, pages 895-900, 2006.

[22] R. A. Knepper and M. T. Mason. Empirical sampling of path sets for local area motion planning. submitted to the 11th International Symposium on Experimental Robotics, 2008.

[23] R. Murray, Z.X. Li, and S. Sastry. A Mathematical Introduction to Robotic Manipulation. CRC Press, 1994. 